\title{
Functional outcomes in geriatric patients with spinal cord injuries at a tertiary care rehabilitation hospital in Saudi Arabia
}

\author{
Sami Ullah ${ }^{1} \cdot$ Irfan Qamar $^{2} \cdot$ Ahmad Zaheer Qureshi $^{1} \cdot$ Amani Abu-Shaheen $^{3} \cdot$ Asim Niaz $^{4}$
}

Received: 3 December 2017 / Revised: 22 June 2018 / Accepted: 22 June 2018

(c) International Spinal Cord Society 2018

\begin{abstract}
Study design Retrospective study

Objective To identify demographic features, clinical characteristics, and complications associated with spinal cord injuries/ disorders (SCI/D) among elderly individuals at a rehabilitation hospital and to measure the functional outcomes of rehabilitation.

Setting Rehabilitation hospital in King Fahad Medical City (KFMC), Riyadh, Saudi Arabia.

Methods The study was conducted in elderly individuals (aged $\geq 65$ years) with SCI/D, admitted to an inpatient rehabilitation program between October 2014 and 2015. Demographic and clinical data were recorded along with functional independence measure (FIM) score at admission (FIMa) and discharge (FIMd). Data were descriptively analyzed. Association of non-metric and metric variables with complications was measured using $\chi^{2}$, and Student's $t$-test, respectively.

Results Twenty-four individuals with SCI/D (95.8\% were male and retired) with mean (standard deviation, SD) age of 72.3 (6.3) years were included. The most common co-morbidities were hypertension (75.0\%), and diabetes mellitus (58.3\%). Degenerative cervical myelopathy (33.3\%) was the most common cause of SCD. Of all, nine (37.5\%) individuals had clinical complications (urinary tract infection(UTI); 8/9, surgical wound infection; 1/9). Mean (SD) hospitalization period during inpatient rehabilitation was 66.0 (13.9) days. Mean (SD) FIMa scores improved from 71.7 (17.3) to 85.3 (16.8) at discharge. Co-morbidities associated with complications were peripheral vascular disease, ischemic heart disease, and stroke.

Conclusion In Saudi Arabia, non-traumatic spinal etiologies are the most frequent cause of spinal cord dysfunction in the elderly. Male gender, hypertension, and diabetes mellitus were high-risk factors among the geriatric age group with SCI/D. Elderly individuals with SCI/D without complications can have a shorter hospitalization period and higher functional gains during rehabilitation.
\end{abstract}

Sami Ullah

drsamipmr@gmail.com

1 Department of Physical Medicine and Rehabilitation, Rehabilitation Hospital, King Fahad Medical City, Riyadh, Saudi Arabia

2 General Internal Medicine and Respiratory Medicine, Eastbourne District General Hospital, Eastbourne, UK

3 Research Center, King Fahad Medical City, Riyadh, Saudi Arabia

4 Department of Physical Medicine and Rehabilitation, King Fahad Specialist Hospital, Dammam, Saudi Arabia

\section{Introduction}

Spinal cord injuries (SCI) and spinal cord disorders (SCD) can cause devastating neurological damage, affecting nearly all systems of the human body [1]. Although SCI is most commonly observed among young individuals; studies have reported that the geriatric population is the second most affected group [2-5]. The geriatric population is more vulnerable to SCD (degenerative cervical myelopathy, lumbar canal stenosis, vertebral tuberculosis, and vertebral osteomyelitis) owing to age-related co-morbidities, reduced independence, and poor tolerance to immobilization. These factors may obscure the rehabilitation in elderly individuals with SCI/D [6-8]. The leading cause of SCI among the geriatric population is falls, followed by motor vehicle accidents [9]. 
One of the prognostic factors for better functional outcome in individuals with SCI/D is an early rehabilitation. Performance and function of an individual declines with advancing age. Rehabilitation interventions, in a specialized setup, should be initiated as early as possible, as delays may lead to detrimental effects on the functional outcomes [10, 11]. Management of the geriatric SCI/D population should be undertaken with an understanding of the individual's unique pathophysiology and concerns associated with their age-related co-morbidities.

Although, the clinical characteristics of elderly individuals with SCI/D have been mentioned in the literature, the findings have been inconsistent [12, 13]. Thus, geriatric $\mathrm{SCI} / \mathrm{D}$ is an essential health issue that warrants more consideration than previously established. Moreover, as compared to developed countries, Saudi Arabia has undergone limited research focusing on the geriatric population with SCI/D. The present study was conducted to identify the demographic profile, clinical characteristics, and complications associated with SCI/D among a geriatric population at a rehabilitation hospital in Riyadh-Saudi Arabia. Rehabilitation outcomes among the same population were also investigated.

\section{Material and methods}

\section{Study design}

This retrospective chart review was carried out at a rehabilitation hospital in King Fahad Medical City (KFMC), Riyadh, Saudi Arabia. All the individuals with SCI/D in the geriatric age group (aged 65 years or above; cutoff used by the United States Census Bureau to define the elderly population) who were admitted to our SCI rehabilitation program from October 2014 to 2015, were included in the study [14]. At KFMC, individuals with SCI/D underwent a comprehensive, integrated inpatient rehabilitation program (CIIRP) meeting the standards of commission on accreditation of rehabilitation facilitates (CARF) [15]. The SCI rehabilitation program has both inpatient and outpatient services. Moreover, it receives referrals from all regions of the country including internal transfers from the hospital. Individuals with SCI/D are admitted after their acute treatment and of these individuals, many have chronic complications.

Demographic and clinical data collected included age, gender, employment status, discharge destination, co-morbidities, cause of injury, length of hospital stay, and complications (adverse effects of an existing condition i.e., complications of SCI/D. Co-morbidities were defined as health conditions, other than SCI/D and its evident consequence, recognized and treated before the recent admission. The functional independence measure score (FIM) was recorded at admission (FIMa), and discharge (FIMd). A higher FIM score meant a greater level of independence.

\section{Statistical analysis}

Data were described as mean (standard deviation, SD) and/ or percentages. Functional outcome and other factors associated with the outcome were represented by presence or absence of complications in the final outcome variable. Association of non-metric variables with complications was measured by $\chi^{2}$-test, and Student's $t$-test was applied for metric variables. The data analysis was performed using SPSS 22.0 software (SPSS Inc., Chicago, IL, USA).

\section{Results}

Overall, 24 individuals with SCI/D, with mean (SD) age of 72.3 (6.3) years (age range: 66-90 years), were included in the study. Twenty-three $(95.8 \%)$ of them had retired from work and were male. All participants had been discharged from the hospital. The most common co-morbidities observed included hypertension $(n=18 ; 75.0 \%)$, diabetes mellitus $(n=14 ; 58.3 \%)$, and deep vein thrombosis $(n=3$; $12.5 \%)$. Non-traumatic SCD were observed in $19(79.17 \%)$ patients while $5(20.83 \%)$ patients suffered with traumatic SCI. Degenerative cervical myelopathy $(n=8 ; 33.3 \%)$ was the most common cause of non-traumatic SCI, followed by lumbar canal stenosis $(n=7 ; 29.2 \%)$, whereas traffic accidents $(n=4 ; 16.7 \%)$ was the most common cause of traumatic SCI. Nine $(37.5 \%)$ participants experienced clinical complications, of whom eight $(33.3 \%)$ had a UTI and one $(4.2 \%)$ was suffering with surgical wound infection. The mean (SD) length of hospital stay during inpatient rehabilitation was 66.0 (3.9) days (Table 1). Moreover, the mean (SD) FIM scores were 71.7 (17.3) at the time of admission and improved to 85.3 (16.8) by the time of discharge. Individuals without complications during inpatient rehabilitation stayed in the hospital for a significantly shorter mean length $(p<0.02)$, and had a higher score for FIM at admission $(p<0.02)$ and at discharge $(p<0.03)$ (Table 2$)$. The top five co-morbidities that were found to be associated with complications were peripheral vascular disease, ischemic heart disease, stroke, hypertension, and diabetes mellitus.

\section{Discussion}

The Kingdom of Saudi Arabia is the largest Middle East country [16]. SCI, being one of the commonly observed 
Table 1 Demographic profile, clinical characteristics and complications during rehabilitation of the study participants

\begin{tabular}{|c|c|}
\hline Variables & $N(\%)$ \\
\hline \multicolumn{2}{|l|}{ Gender } \\
\hline Male & $23(95.8)$ \\
\hline Female & $1(4.2)$ \\
\hline \multicolumn{2}{|l|}{ Employment status } \\
\hline Employed & $1(4.2)$ \\
\hline Retired & $23(95.8)$ \\
\hline \multicolumn{2}{|l|}{ Discharge destination } \\
\hline Home & $24(100)$ \\
\hline Long-term facility & $0(0)$ \\
\hline Acute care transfer & $0(0)$ \\
\hline \multicolumn{2}{|l|}{ Co-morbidities } \\
\hline Hypertension & $18(75.0)$ \\
\hline Diabetes mellitus & $14(58.3)$ \\
\hline Deep vein thrombosis & $3(12.5)$ \\
\hline Stroke & $2(8.3)$ \\
\hline Renal disease & $2(8.3)$ \\
\hline Ischemic heart disease & $2(8.3)$ \\
\hline Asthma & $1(4.2)$ \\
\hline Parkinson's disease & $1(4.2)$ \\
\hline Peripheral vascular disease & $1(4.2)$ \\
\hline Pulmonary embolism & $1(4.2)$ \\
\hline \multicolumn{2}{|l|}{ Complications during rehabilitation } \\
\hline Urinary tract infection & $8(33.3)$ \\
\hline Surgical wound infection & $1(4.2)$ \\
\hline No complications & $15(62.5)$ \\
\hline \multicolumn{2}{|l|}{ Cause of injury } \\
\hline \multicolumn{2}{|l|}{ Traumatic } \\
\hline Traffic accidents & $4(16.7)$ \\
\hline Fall from height & $1(4.2)$ \\
\hline \multicolumn{2}{|l|}{ Non-traumatic } \\
\hline Degenerative cervical myelopathy & $8(33.3)$ \\
\hline Lumbar canal stenosis & $7(29.2)$ \\
\hline Vertebral tuberculosis & $3(12.5)$ \\
\hline Vertebral osteomyelitis & $1(4.2)$ \\
\hline Length of hospital stay (days) & $66.0 \pm 13.9$ \\
\hline
\end{tabular}

Data presented either as number and percentage or as mean and standard deviation

medical conditions in Saudi Arabia, requires adequate management. It warrants intensive and customized rehabilitation plans to improve functional outcomes of the persons with SCI [17]. Various epidemiological studies, functional outcome related research, and narrative reviews published in the last decade, have focused on traumatic SCI in Saudi Arabia [17-19]. A recent study conducted in Saudi Arabia by Mahmoud et al., 2017 described functional status, length of stay, and rehabilitation trends in adults with SCI (mean age: $35 \pm 17$ years) [20]. However, none of the recently
Table 2 Length of hospital stay and functional independence measure scores by complications during rehabilitation

\begin{tabular}{llll}
\hline & Complications* & Mean \pm SD & $p$-value \\
\hline LOS (days) & - & $45.8 \pm 7.6$ & 0.021 \\
& + & $99.7 \pm 33.1$ & \\
FIMa & - & $77.5 \pm 5.0$ & 0.021 \\
& + & $62.0 \pm 2.0$ & \\
FIMd & - & $91.1 \pm 4.6$ & 0.035 \\
& + & $75.7 \pm 3.1$ & \\
\hline
\end{tabular}

* Absent (-), Present (+)

LOS Length of hospital stay, FIMa Functional independence measure at the time of admission, FIMd Functional independence measure time of discharge

conducted studies focused on the same in geriatric population. This is the first study of its kind in the Arabian Peninsula and was conducted in the largest ministry of health rehabilitation hospital in the country. It examines the moderating effects of old age on the various aspects related to SCI, i.e., co-morbidities, etiology, complications, and functional outcome in a rehabilitation setting.

There are several trends which are prevalent in geriatric $\mathrm{SCI} / \mathrm{D}$ and its treatment outcome. In this study, the mean (SD) age of individuals with SCI was 72.3 (6.3) years; the majority of them were male and were retired from work. Women are potentially at lower risk than men for SCI, as evidenced by the previous literature on SCI in the Kingdom [17-20]. These findings may be explained by the fact that women work in activities which are less commonly associated with a risk of injury. In Saudi Arabia, at the time of study, there were no driving privileges for women and women were usually not involved in manual labor jobs.

There is a strong association between the age above 64 and the occurrence of co-morbidities. A retrospective study conducted by Krassioukov et al., 2003 compared 30 young (age 17-56 years) individuals with SCI and 28 elderly individuals (age 60-89 years) with SCI and reported the comorbidities to be more associated with the elderly $(p<0.01)$ along with higher incidence of complications observed in the elderly (elderly vs. young: $57.1 \%$ vs. $33.3 \% ; p=0.11$ ) [21]. In the present study as well, the elderly population with SCI were associated with co-morbidities like diabetes mellitus, hypertension, deep vein thrombosis, renal disease, and ischemic heart disease.

In the present study, SCI/D and unknown etiologies were all included to assess the epidemiological characteristics and etiologies. The most common cause of SCD was nontraumatic while only four cases were reported due to a fall. In contrast, previous studies conducted by Kannus et al. and Hagen et al. reported fall as a main cause of SCI amongst the older population $[22,23]$. Other studies reported highenergy injuries such as traffic accidents, high falls, and 
being struck by falling objects to be responsible for SCI in younger population (21-60 years), while low-energy injuries such as low falls were the most common cause of SCI for the elderly population over 60 years old $[24,25]$. To date, no studies have focused on the geriatric population with non-traumatic SCI in Saudi Arabia. In this study, the most common cause of SCD was degenerative cervical myelopathy while traffic accidents was the comments cause of traumatic SCI.

In a study carried out in Saudi Arabia, which included 1340 patients with spinal disorders of lumbar and cervical region, $39.7 \%$ of the patients were 50 years of age and above, including $3 \%$ of patients with age $>70$ years [26]. Most of the patients were in the age range of 30-49 years. The frequency of spondylosis was higher (37.2\%) as compared to disc disease (32.6\%). About $42 \%$ of the patients, in the age group between 30 and 49 years, were reported to have spondylosis. This suggests a reason to investigate early spondylosis in the Saudi population, as it may correlate to potentially serious outcomes with advancing age, like SCD.

Chronic complications in elderly persons with SCD include osteoporosis and bone fracture, pressure ulcers, pain syndrome, thromboembolic disease, musculoskeletal, and metabolic complications. Limited data identifies complication during inpatient rehabilitation for individuals with SCI in a geriatric population [27]. The present study included only new complications, that occurred during the rehabilitation stay. UTI was the most common complication encountered during inpatient rehabilitation, followed by surgical site infection. Other studies report chest infections and pressure ulcers as well. In our data no one had chest infections or venous thromboembolism, while only one individual had a pressure ulcer. A retrospective study conducted in China by Yang et al. on 3832 individuals with SCI reported pulmonary infections, UTIs, and bedsores as the major complications [25]. In addition, Pagliacci et al. conducted a prospective, multi-centered follow-up observational study to evaluate survival, complications, readmissions, and maintenance of clinical outcome in 511 individuals with SCI and reported urinary tract infections, pain, spasms, and bedsores as the main four complications of SCI [28]. Complications associated with geriatric SCI are generally preventable. Hence, efforts should be focused on preventive measures, given that elderly population is at high risk of acquiring medical complications and rehospitalization.

In a geriatric group of individuals with SCI who underwent an inpatient rehabilitation program, an increase in FIM scores, by an average of 29 points from the time of admission to discharge, was reported by Joseph et al.[29]. The present study reports FIM gain score of 17 and 13 in geriatric patients without and with complications, respectively. It is also important to note that FIMa of patients with complications (62.0) was lower as compared to FIMa of patients without complications (77.5). Moreover, patients with low FIMa were likely to have low FIM gains, as compared to patients without complications. Results also reveal that individuals without complications during inpatient rehabilitation were found to have a shorter hospitalization period and higher FIMa and FIMd scores. This is consistent with other studies reported in SCI where Eastwood et al. also found that SCI with complications may lower FIM scores at discharge [30]. Cardenas et al. also reported that the continuum of care for individuals with SCI is affected by medical complications [31]. The complications associated with geriatric SCI can be prevented by taking appropriate measures. Furthermore, high functional independence could be achieved with shorter hospitalization period.

Al-Jadid MS reports that $\sim 135,000$ or nearly $0.8 \%$ of the total Saudi population has a disability while SCI is one of leading cause of disability in the Kingdom [32]. He also highlights that disability is one of the most imperative social and economic medical issues. Despite a higher disability rate due to $\mathrm{SCI}$, the literature on $\mathrm{SCI} / \mathrm{D}$ in a geriatric population in Saudi Arabia is limited.

Limitations to our study include a small study sample size. Moreover, we did not have any control group such as a geriatric population without SCI. Additionally, the level of SCI was not assessed using AIS score.

Future studies should be conducted to determine the functional outcomes in elderly with SCI. This will help in improving the admission criteria, rehabilitation plan and strategies to prevent complications of SCI.

\section{Conclusion}

In a geriatric population with SCI treated in the specialized rehabilitation center in Riyadh, Saudi Arabia a nontraumatic etiology of SCI is dominating, most of the patients are males, and most frequent co-morbidities associated with the risk of complications are hypertension and diabetes mellitus. Having no complications was related to lower length of hospital stay and higher functional gain during rehabilitation. Elderly with SCI can benefit from inpatient rehabilitation program, with special emphasis on prevention of complications. Rehabilitation needs to be promoted as an integral intervention during the acute phase of care and can help to improve functional outcomes in the geriatric population. Diagnosis of early spondylosis and degenerative disc disease need to be considered, to avoid potential serious outcomes with advancing age; however, it requires larger studies to strengthen the evidence. 


\section{Compliance with ethical standards}

Ethical considerations This study was approved by the Ethics Committee at KFMC, Riyadh, Saudi Arabia.

Conflict of interest The authors declare that they have no conflict of interest.

\section{References}

1. Wyndaele M, Wyndaele JJ. Incidence, prevalence and epidemiology of spinal cord injury: what learns a worldwide literature survey? Spinal Cord. 2006;44:523-9.

2. National Spinal Cord Injury Statistical Centre: Annual Statistics Report. 2008 [cited 2017 October 4]. Available at www.nscisc.ua b.edu.

3. Pickett GE, Campos-Benitez M, Keller JL, Duggal N. Epidemiology of traumatic spinal cord injury in Canada. Spine. 2006;31:799-805.

4. Jabbour P, Fehlings M, Vaccaro AR, Harrop JS. Traumatic spine injuries in the geriatric population. Neurosurg Focus. 2008;25: E16.

5. Lenehan B, Street J, Kwon BK, Noonan V, Zhang H, Fisher CG, et al. The epidemiology of traumatic spinal cord injury in British Columbia, Canada. Spine. 2012;37:321-9.

6. Smith S, Purzner T, Fehlings M. The epidemiology of geriatric spinal cord injury. Top Spinal Cord Inj Rehabil. 2010;15:54-64.

7. Fassett DR, Harrop JS, Maltenfort M, Jeyamohan SB, Ratliff JD, Anderson DG, et al. Mortality rates in geriatric patients with spinal cord injuries. J Neurosurg Spine. 2007;7:277-81.

8. Kennedy P, Evans MJ, Berry C, Mullin J. Comparative analysis of goal achievement during rehabilitation for older and younger adults with spinal cord injury. Spinal Cord. 2003;41:44-52.

9. Chen Y, Tang Y, Allen V, DeVivo MJ. Aging and spinal cord injury: external causes of injury and implications for prevention. Top Spinal Cord Inj Rehabil. 2015;21:218-26.

10. Groah SL, Charlifue S, Tate D, Jensen MP, Molton IR, Forchheimer M, et al. Spinal cord injury and aging: Challenges and recommendations for future research. Am J Phys Med Rehabil. 2012;91:80-93.

11. National Institute on Aging. Biology of aging: research today for a healthier tomorrow. Baltimore: National Institute on Aging, National Institute of Health, U.S. Department of Health and Human Services. 2011 [cited 2017 January 27]. Available from http://www.nia.nih.gov/sites/default/files/biology_on_aging.pdf.

12. Furlan JC, Bracken MB, Fehlings MG. Is age a key determinant of mortality and neurological outcome after acute traumatic spinal cord injury? Neurobiol Aging. 2010;31:434-46.

13. Scivoletto G, Morganti B, Ditunno P, Ditunno JF, Molinari M. Effects on age on spinal cord lesion patients' rehabilitation. Spinal Cord. 2003;41:457-64.

14. U.S.-Census-Bureau. Sixty-Five Plus in the United States. 2011 [cited 2017 October 4]. Available from http://www.census.gov/ population/socdemo/statbriefs/agebrief.html.
15. Comprehensive Integrated Inpatient Rehabilitation Programs. 2017 [cited 2017 October 4]. Available from http://www.carf.org/ Programs/ProgramDescriptions/MED-Inpatient-Rehab-Hospital/.

16. Health Statistical Year Book. Kingdom of Saudi Arabia. 2011 [cited 2018 January 9]. Available from https://www.moh.gov.sa/ en/Ministry/Statistics/book/Documents/1433.pdf.

17. Robert AA, Zamzami MM. Traumatic spinal cord injury in Saudi Arabia: a review of the literature. Pan Afr Med J. 2013;16:104.

18. Alshahri SS, Cripps RA, Lee BB, Al-Jadid MS. Traumatic spinal cord injury in Saudi Arabia: an epidemiological estimate from Riyadh. Spinal Cord. 2012;50:882-4.

19. Abdul-Sattar AB. Predictors of functional outcome in patients with traumatic spinal cord injury after inpatient rehabilitation: in Saudi Arabia. NeuroRehabilitation. 2014;35:341-7.

20. Mahmoud H, Qannam H, Zbogar D, Mortenson B. Spinal cord injury rehabilitation in Riyadh, Saudi Arabia: time to rehabilitation admission, length of stay and functional independence. Spinal Cord. 2017;5:509.

21. Krassioukov AV, Furlan JC, Fehlings MG. Medical co-morbidities, secondary complications, and mortality in elderly with acute spinal cord injury. J Neurotrauma. 2003;20:391-9.

22. Kannus P, Niemi S, Palvanen M, Parkkari J. Continuously increasing number and incidence of fall-induced, fracture associated, spinal cord injuries in elderly persons. Arch Intern Med. 2000;160:2145-9.

23. Hagen EM, Aarli JA, Gronning M. The clinical significance of spinal cord injuries in patients older than 60 years of age. Acta Neurol Scand. 2005;112:42-47.

24. McDonald JW, Sadowsky C. Spinal-cord injury. Lancet. 2002;359:417-25.

25. Yang R, Guo L, Wang P, Huang L, Tang Y, Wang W, et al. Epidemiology of spinal cord injuries and risk factors for complete injuries in guangdong, china: a retrospective study. PLoS ONE. 2014;9:e84733.

26. Alshami AM. Prevalence of spinal disorders and their relationships with age and gender. Saudi Med J. 2015;36:725-30.

27. Hagen EM. Acute complications of spinal cord injuries. World $\mathbf{J}$ Orthop. 2015;6:17-23.

28. Pagliacci MC, Franceschini M, Di Clemente B, Agosti M, Spizzichino L. A multicentre follow-up of clinical aspects of traumatic spinal cord injury. Spinal Cord. 2007;45:404-10.

29. Joseph B, Pandit V, Aziz H, et al. Rehabilitation after trauma; does age matter? J Surg Res. 2013;184:541-5.

30. Eastwood EA, Hagglund KJ, Ragnarsson KT, Gordon WA, Marino RJ. Medical rehabilitation length of stay and outcomes for persons with traumatic spinal cord injury-1990-7. Arch Phys Med Rehabil. 1999;80:1457-63.

31. Cardenas DD, Hoffman JM, Kirshblum S, McKinley W. Etiology and incidence of rehospitalization after traumatic spinal cord injury: a multicenter analysis. Arch Phys Med Rehabil. 2004;85:1757-63.

32. Al-Jadid MS. Disability in Saudi Arabia. Saudi Med J. 2013;34:453-60. 